\title{
Nulls and Side lobe Levels Control in a Time Modulated Linear Antenna Array by Optimizing Excitations and Element Locations Using RGA
}

\author{
Bipul Goswami and Durbadal Mandal \\ Department of Electronics and Communication Engineering \\ National Institute of Technology Durgapur \\ West Bengal, India- 713209 \\ Email: \{durbadal.bittu, goswamibipul\}@gmail.com
}

\begin{abstract}
In this paper a novel approach based on the Real coded Genetic Algorithm (RGA) is proposed to improve nulling performance as well as suppression of Side Lobe Level (SLL) of a Time Modulated Linear Antenna Arrays (TMLA). RGA adjusts the static excitation amplitudes as well as the location of each element from the origin to place deeper nulls in the desired direction. Three design examples are presented that illustrate the use of the RGA, and the optimization goal in each example is easily achieved.
\end{abstract}

Index terms - Time Modulated Linear Antenna Array; Far-field Pattern; Genetic Algorithm; Amplitude Excitation; Deeper Null; Side Lobe Level.

\section{INTRODUCTION}

Antenna Array is formed by assembling the radiating elements in a particular electrical or geometrical configuration. In most cases the elements are identical. Total field of the antenna array is found by vector addition of the fields radiated by each individual element. There are five control parameters in an antenna array that can be used to shape the pattern properly, they are, the geometrical configuration (linear, circular, rectangular, spherical etc.) of the overall array, relative displacement between elements, excitation amplitude of the individual elements, excitation phase of the individual elements and relative pattern of the individual elements [1-4]. In many communication applications it is required to design a highly directional antenna. Antenna Arrays have high gain and directivity compared to an individual radiating element.

As compared to conventional antenna arrays, the time modulated antenna arrays introduce a fourth dimension 'time' into the design [5-13]. By using the additional degree of freedom, time, more specifically, by properly turning on and off the array elements according to suitable time sequences, the stringent requirements on various error tolerances can be relaxed to the levels of those in conventional SLLs. However the major disadvantage of time modulated array is that, there are many 
sideband signals spaced at multiples of modulation frequency, which implies that part of electromagnetic energy radiated or received by the antenna array was shifted to the sidebands.

The increasing pollution of electromagnetic environment has prompted the study of array pattern nulling techniques. These techniques are very important in radar, sonar and communication systems for minimizing degradation in signal to noise ratio performance due to undesired interference [3-4]. A lot of research works are going on for reducing the signal at nulls and Side Lobe Levels [5-20]. Time modulated antenna arrays are attractive for the synthesis of low/ultralow side lobes [6-7, 11-13]. In $[6-7,11]$, a differential evolution algorithm has been used to optimize the static-mode coefficients as well as the durations of the time pulses leading to a significant reduction of the sideband level. An approach to induce deeper nulls by controlling excitations amplitude of antenna elements in a time modulated array using Genetic Algorithm has been proposed in [8]. An approach for estimating direction of arrivals (DOA) in TMLAs with unidirectional phase center motion (UPCM) scheme is proposed in [9].

Design goal of this paper is to introduce deeper null/nulls to some desired direction and to suppress the relative SLL with respect to main beam for a TMLA of isotropic elements. This is done by designing the relative spacing between the elements, with a non-uniform excitation over the array aperture. This involves non-monotonic variation in antenna element parameters, which becomes a highly complex optimization problem. The classical optimization methods cannot bear the demand of such complex optimization problem.

Classical optimization methods have several disadvantages such as: i) highly sensitive to starting points when the number of solution variables and hence the size of the solution space increase, ii) frequent convergence to local optimum solution or divergence or revisiting the same suboptimal solution, iii) requirement of continuous and differentiable objective cost function (gradient search methods), iv) requirement of the piecewise linear cost approximation (linear programming), and v) problem of convergence and algorithm complexity (non-linear programming). For the optimization of such complex, highly non-linear, discontinuous, and non-differentiable array factors; various heuristic search evolutionary techniques were adopted. In this paper, the evolutionary technique, Real coded Genetic Algorithm (RGA) [3-4, 21-23] is used to get the desired pattern of the array. The time modulation period is divided into numerous minimal time steps with the same length, where the ONOFF status for each time step is followed by a scheme given in the paper.

A broadside uniform linear array with uniform inter-element spacing is considered for reference. The array is symmetric with respect to the origin with equal spacing between any two consecutive elements. The phase difference between any two elements is kept zero. The RGA adjusts the excitation coefficients and location of elements from array centre for imposing deeper nulls at predefined directions. A cost function is defined which helps to keep the nulls and side lobes at lower levels. 
The rest of the paper is arranged as follows: in section II, the general design equations for the nonuniformly excited and unequally spaced linear antenna array are stated. Then, in section III, a brief introduction for the Genetic Algorithm is presented. Numerical simulation results are presented in section IV. Finally the paper concludes with a summary of the work in section V.

\section{DESIGN EQUATIONS}

A broadside linear array of $2 \mathrm{M}$ isotropic radiators [1-2] is considered as shown in Figure 1, in which each element is controlled by a high speed RF switch and excited with non uniform current excitation. The array elements are assumed to be uncoupled and having equal inter-elements spacing along the z-axis with its centre at the origin. The array is symmetric in both, geometry and excitation with respect to the array centre. Only Amplitude excitations and spacing between each element are used to change the antenna pattern.

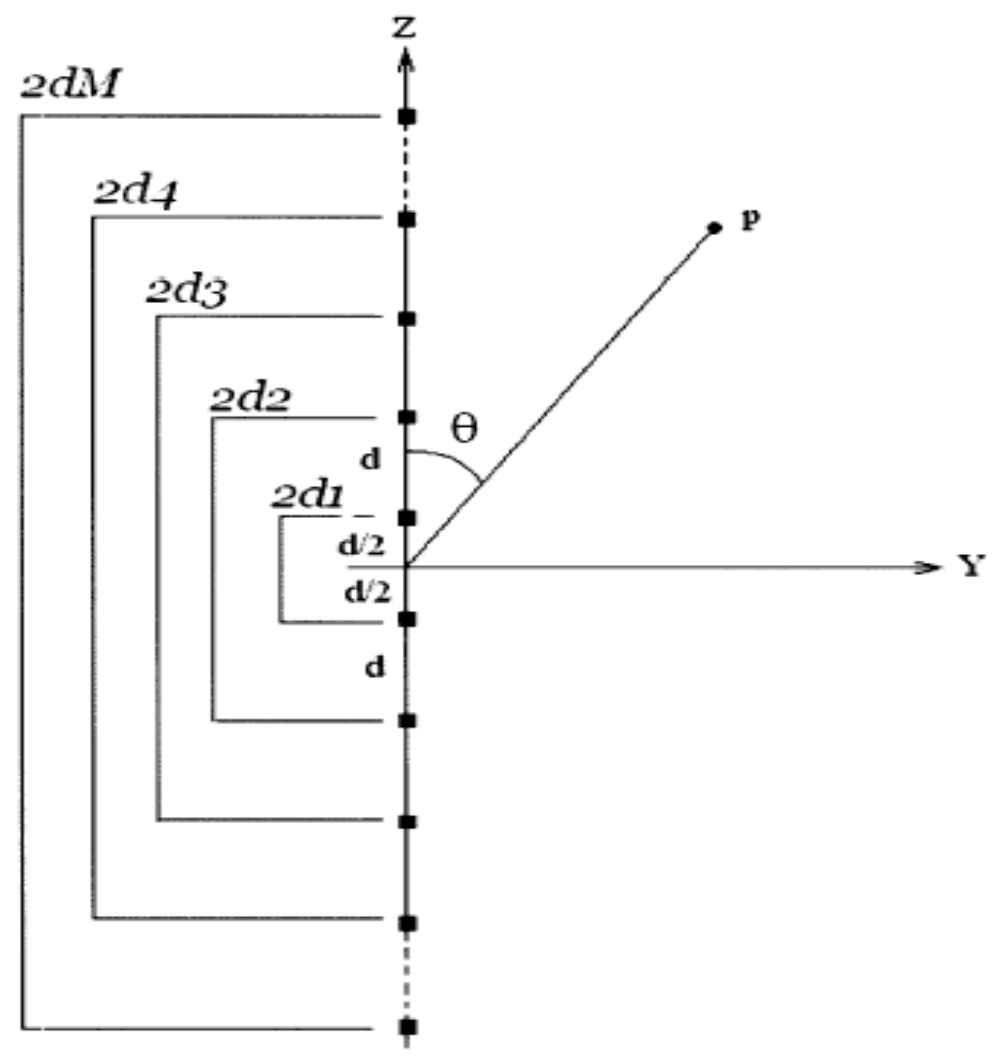

Fig. 1. Geometry of a $2 \mathrm{M}$ element symmetric linear array along the $\mathrm{z}$ axis.

The free space far-field pattern [1-2] in azimuth plane ( $x-y$ plane) with symmetric amplitude distributions is given by (1):

$$
A F(I, \varphi, d, t)=2 \sum_{n=1}^{M} U_{n}(t) I_{n} \cos \left[k X_{n} \cos (\theta)+\varphi_{n}\right]
$$


where, $\theta$ denotes the angle measured from the broadside direction of the array. $I_{n}, \varphi_{n}$ and $X_{n}$ are respectively, current excitation amplitude, excitation phase, and location of the $\mathrm{n}^{\text {th }}$ element from the origin. In this paper $\varphi_{n}$ is kept as zero. $k=2 \pi / \lambda$, the propagation constant where $\lambda$ being the signal wave length, $d$ is the spacing between elements, $2 M$ is total number of elements in the array. $U_{n}(t)=$ periodic switch ON-OFF time sequences function for $\mathrm{n}^{\text {th }}$ where each element is switched ON for $\tau$, where $0 \leq \tau \leq \mathrm{T}$.

The array elements are numbered 1 to $\mathrm{M}$ from the origin in a symmetric array where total numbers of elements are $2 \mathrm{M}$. Firstly the left most $\mathrm{P}$ elements $(\mathrm{P}<\mathrm{M})$ are turned on for time step $\tau$, where

$$
\tau=\frac{T}{M-P+1}
$$

Here $\mathrm{T}$ is the time modulation period. In first time step, the $P$ consecutive elements numbered from 1 to $P$ are switched $\mathrm{ON}$. In the next time step, 2 to $P+1$ are switched $\mathrm{ON}$ and so on. In this work $U_{n}(t)$ is taken as [6-7]:

$$
U_{n}(t)=\left\{\begin{array}{lc}
1, & \varepsilon_{1} \tau \leq t \leq \varepsilon_{2} \tau \\
0, & \text { otherwise }
\end{array}\right.
$$

where

$$
\begin{aligned}
& \varepsilon_{1}=\left\{\begin{array}{cc}
0, & n \leq P \\
n-P, & \text { otherwise }
\end{array}\right. \\
& \varepsilon_{2}=\left\{\begin{array}{cc}
n, & n \leq M-P+1 \\
M-P+1, & \text { otherwise }
\end{array}\right.
\end{aligned}
$$

To illustrate the $U_{n}(t)$ in detail, an example of a time modulated linear array with $M=8$ and $P=4$ is shown in Fig. 2. 


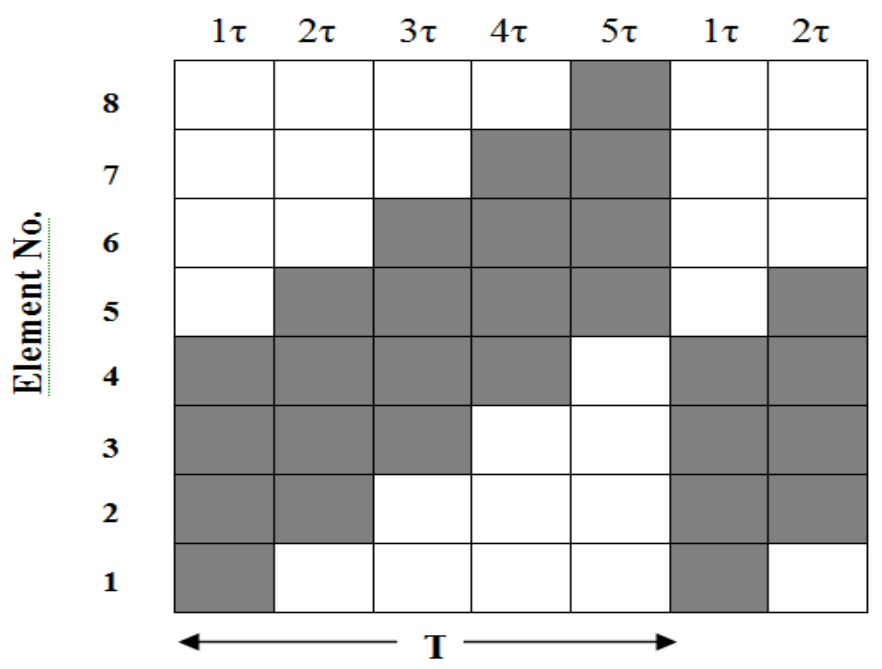

Fig. 2. Switch on time sequences of a 16 element $(M=8)$ TMLA with $\mathrm{P}=4$.

After defining the far-field radiation pattern, the next step in the design process is to formulate the objective function which is to be minimized.The objective function "Cost Function" $(C F)$ or Fitness function which to be minimized with RGA for introducing deeper null is given in (6).

$$
C F=C_{1} \times \frac{\mid \prod_{i=1}^{m} A F\left(\text { null }_{i}\right) \mid}{\left|A F_{\text {max }}\right|}+C_{2} \times \sum_{k=1}^{K} H(k) \times\left(Q_{k}-\delta\right)
$$

Where $m$ is the maximum number of positions where null can be imposed. In this paper, one and two has been considered as the value of ' $m$ '. $A F(n u l l)$ is the array factor value at the particular null position. $A F_{\max }$ is the maximum value of the array factor. The second term in the CF is added to reduce the side lobe up to a desired level. Here $\mathrm{K}$ denotes number of side lobes in the original pattern. $Q_{k}$ is the side lobe level in $\mathrm{dB}$ generated by the individual population at some peak point $\theta_{k}$. $\delta$ is the desired value of the side lobe level in $\mathrm{dB} . H(k)$ is defined as

$$
H(k)= \begin{cases}1, & \left(Q_{k}-\delta\right)>0 \\ 0, & \left(Q_{k}-\delta\right) \leq 0\end{cases}
$$

The side lobes whose peak exceeds threshold $\delta$, that side lobe must be suppressed and for this purpose $H(k)$ is adopted in the "cost function" expression. In cost function, for the first term, both the numerator and denominator are absolute. Further $C_{1}$ and $C_{2}$ are the weighting factors. The weight $C_{1}$ and $C_{2}$ are chosen in such a way that optimization of null remains more dominant, since the prime aim of this work is to achieve deeper null, $C_{1}$ value is taken higher than $C_{2}$. Smaller value of the $C F$ means that array factor values at predefined null positions are less. The RGA technique employed for optimizing current excitation weight and spacing between each elements resulting in the minimization of $C F$. 


\section{EVOLUTIONARY TECHNIQUE: REAL-CODED GENETIC ALGORITHM}

GA is mainly a probabilistic search technique, based on the principles of natural selection and evolution. At each generation it maintains a population of individuals where each individual is a coded form of a possible solution of the problem at hand and called chromosome. Chromosomes are constructed over some particular alphabet, e.g., the binary alphabet $\{0,1\}$, so that chromosomes' values are uniquely mapped onto the decision variable domain. Each chromosome is evaluated by a function known as fitness function, which is usually the fitness function or the objective function of the corresponding optimization problem.

Steps of RGA as implemented for optimization of spacing between the elements and current excitations are [3-4], [18-20]:

- Initialization of real chromosome strings of $n_{p}$ population, each consisting of a set of excitations. Size of the set depends on the number of excitation elements in a particular array design.

- Decoding of strings and evaluation of $C F$ of each string.

- Selection of elite strings in order of increasing $C F$ values from the minimum value.

- Copying of the elite strings over the non-selected strings.

- Crossover and mutation to generate off-springs.

- Genetic cycle updating.

The iteration stops when the maximum number of cycles is reached. The grand minimum $C F$ and its corresponding chromosome string or the desired solution are finally obtained.

The working principle of a GA has been explained briefly in the following figure based on the problem in hand. 


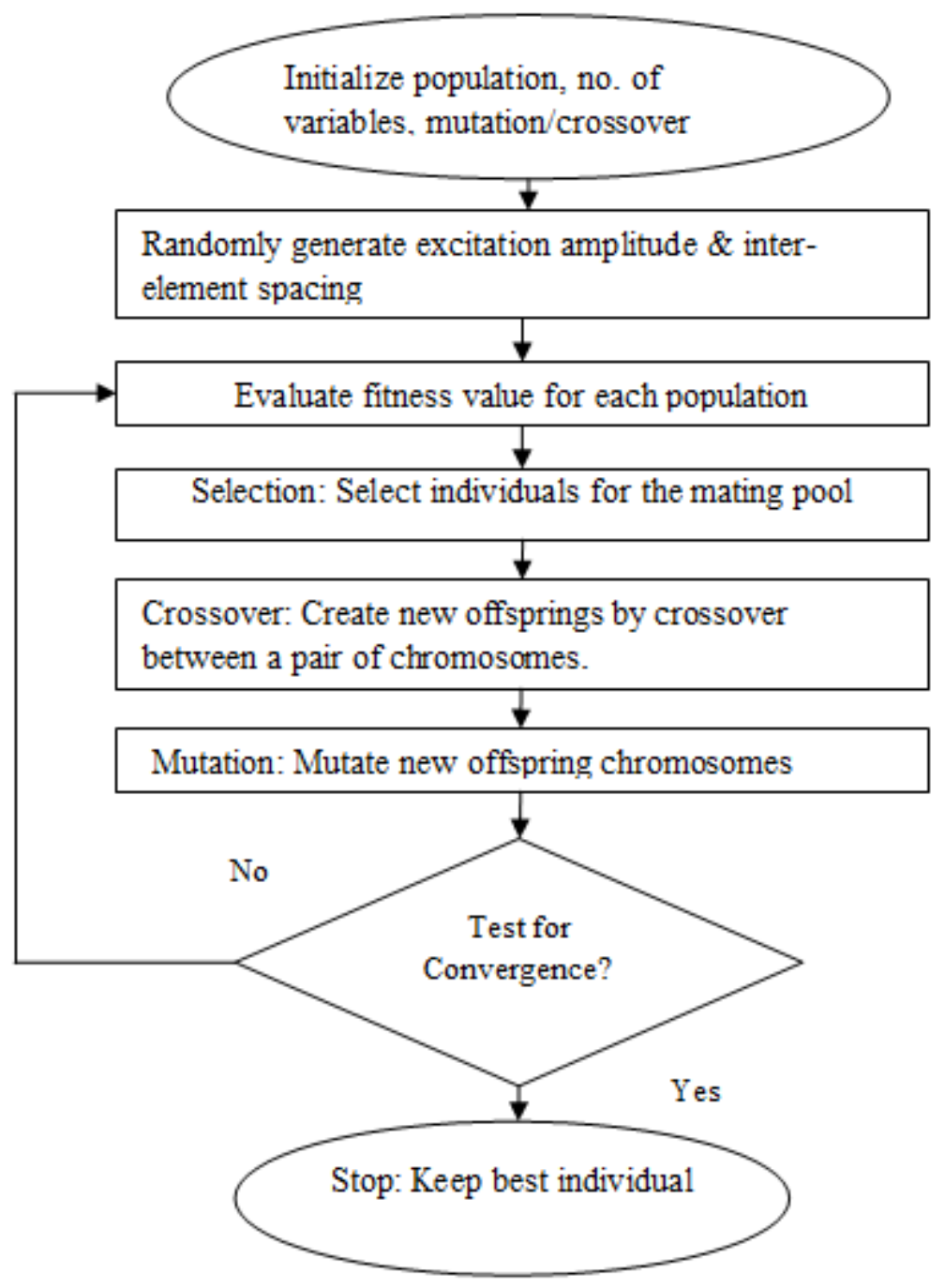

Fig. 3. The GA flow for determining the optimized excitation amplitude and optimum location of array elements.

Desired pattern is generated by jointly optimizing amplitude distributions and location of array elements from the array centre. Here, both excitation amplitude and element location distributions are assumed symmetric with respect to the centre of the array. The chromosomes are corresponded to current excitation weights of antenna elements and location of individual elements from the array centre. Because of symmetry, each chromosome consists of $2 \mathrm{M}$ number of genes where $\mathrm{M}$ is the number of antenna elements on either side of array centre. Here $1^{\text {st }}$ to $\mathrm{M}^{\text {th }}$ genes represent current excitation weights of antenna elements and $(M+1)^{\text {th }}$ to $2 M^{\text {th }}$ genes represent optimal element locations from the array centre. As an example chromosome one $\bar{W}_{1}$ can be represented by (8).

$$
\overline{W_{1}}=\left[W_{11}, W_{12}, \ldots . W_{1 M}, W_{1(M+1)}, W_{1(M+2)}, \ldots, W_{1(2 M)}\right]
$$

Here, $W_{11}, W_{12}, \ldots . W_{1 M}$ are the antenna elements amplitude distributions and $W_{1(M+1)}, W_{1(M+2)}, \ldots, W_{1(2 M)}$ are the locations of array elements from the array centre. Each of this 
current excitation weights and element locations has boundaries with upper and lower limit. The random set of chromosome can be easily constructed using following relation represented by (9).

$$
\bar{W}_{n}=\left(u_{1}-u_{2}\right) \times \bar{r}+u_{2} \quad, \quad u_{2}<\bar{W}_{n} \leq u_{1}
$$

Where, $u_{1}$ and $u_{2}$ are the maximum and minimum limit value of the weights and $\bar{r}$ is real random vector between zero and one. RGA technique generates a set of normalized non-uniform current excitation weights and optimal spacing for all sets of time modulated linear antenna arrays.

\section{NUMERICAL RESULTS}

A 12,16 , and 20 elements linear array of isotropic radiating elements, with $\lambda / 2$ inter-element spacing, is considered for time modulated linear antenna array with $\mathrm{T}=16 \mu s$. The required "switchon" time intervals for each element can be calculated using (2). RGA is applied to get deeper nulls. Every time RGA executed with 400 iterations. The population size was fixed at 120 . For the real coded GA, the mutation probability was set to 0.01 and uniform crossover with crossover probability 1 was taken. RGA algorithm is initialized using random values of excitation $\left(0<I_{n}<1\right)$ and spacing between elements $(\lambda / 2 \leq d<\lambda)$. For predefined nulls of the radiation pattern, the nulling performances are improved. Similarly at predefined peak positions, the nulls are imposed. The programming has been written in Matlab language using MATLAB 7.8.0(R2009a) version on core (TM) 2 duo processor, $3.00 \mathrm{GHz}$ with 2 GB RAM.

The Initial values of maximum SLL and Beam width between first nulls (BWFN) for uniform amplitude excitation $\left(I_{n}=1\right)$ and uniform spacing ( $\lambda / 2$ between adjacent elements) has been given in TABLE I.

TABLE I. SLL AND BWFN For Uniform Excitation $\left(I_{n}=1\right)$ With $\lambda / 2$ InTER-Elements Spacing Linear ARRAY

\begin{tabular}{|c|c|c|c|}
\hline Set No. & Total number of elements (2M) & SLL (dB) & BWFN (degree) \\
\hline I & 12 & -13.06 & 19.10 \\
\hline II & 16 & -13.14 & 14.40 \\
\hline III & 20 & -13.19 & 11.52 \\
\hline
\end{tabular}

Figures 4-6 show the imposing of deeper nulls over $3^{\text {rd }}$ null. For 12, 16, 20-element, the nulls have improved up to $-80 \mathrm{~dB},-80 \mathrm{~dB}$, and $-90 \mathrm{~dB}$ with initial values of $-53.93 \mathrm{~dB},-50.60 \mathrm{~dB},-77.20 \mathrm{~dB}$ respectively. TABLE II shows the resulting amplitude excitation distribution, optimal elements location, initial and final null depth obtained by optimizing the cost function using RGA. In this case the weighting for the array elements $I_{1}, I_{2} \ldots \ldots \ldots . . I_{M}$ are normalized using $\max \left(I_{M}\right)=1$ and 
$X_{1}, X_{2}, \ldots . ., X_{M}$ are normalized using $\lambda / 2$. Figures 4-6 also depict the substantial reductions in maximum peak of the SLL with non-uniform current excitation weights and optimal placement of array elements, as compared to the uniform current excitation weights $\left(I_{M}=1\right)$ and uniform interelements spacing ( $\lambda / 2$ between adjacent elements) case. For example, in optimized pattern the SLL is reduced by more than $1 \mathrm{~dB}$ from $-13.06 \mathrm{~dB}$ down to $-14.27 \mathrm{~dB}$ for 12 elements array. For 16 elements array in optimized pattern SLL is reduced by more than $1 \mathrm{~dB}$ from $-13.14 \mathrm{~dB}$ down to $-14.51 \mathrm{~dB}$. For 20 elements array in optimized pattern SLL is reduced from $-13.19 \mathrm{~dB}$ down to $-14.22 \mathrm{~dB}$. The improved values are shown in TABLE II and TABLE IIA.

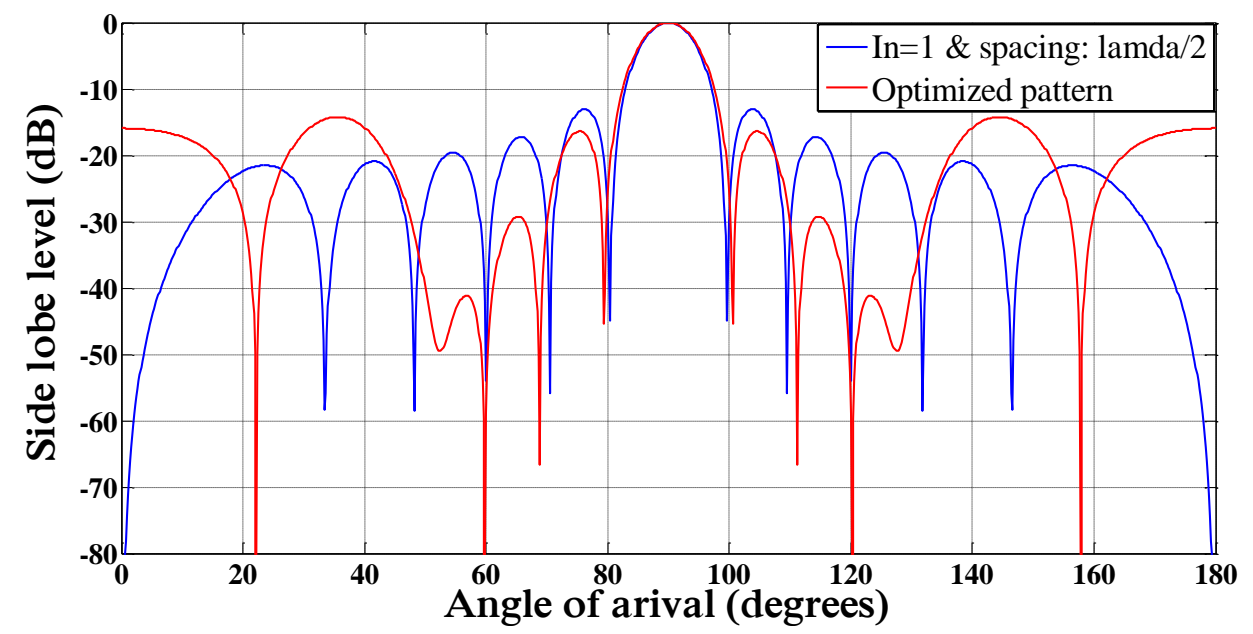

Fig. 4. Best array pattern found by GA for the 12-elements array case with an improved null at 3rd nulls i.e. $\theta=60^{\circ}$ and $\theta=120^{\circ}$.

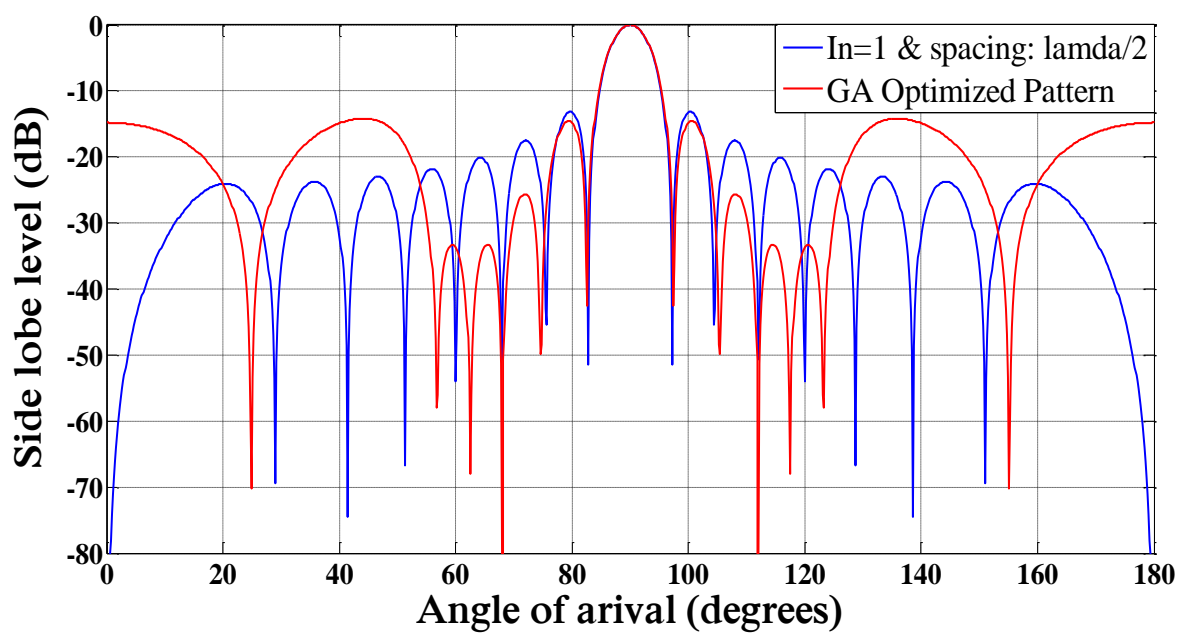

Fig. 5. Best array pattern found by GA for the 16 -elements array case with an improved null at 3rd null i.e. $\theta=68^{0}$ and $\theta=112^{0}$. 


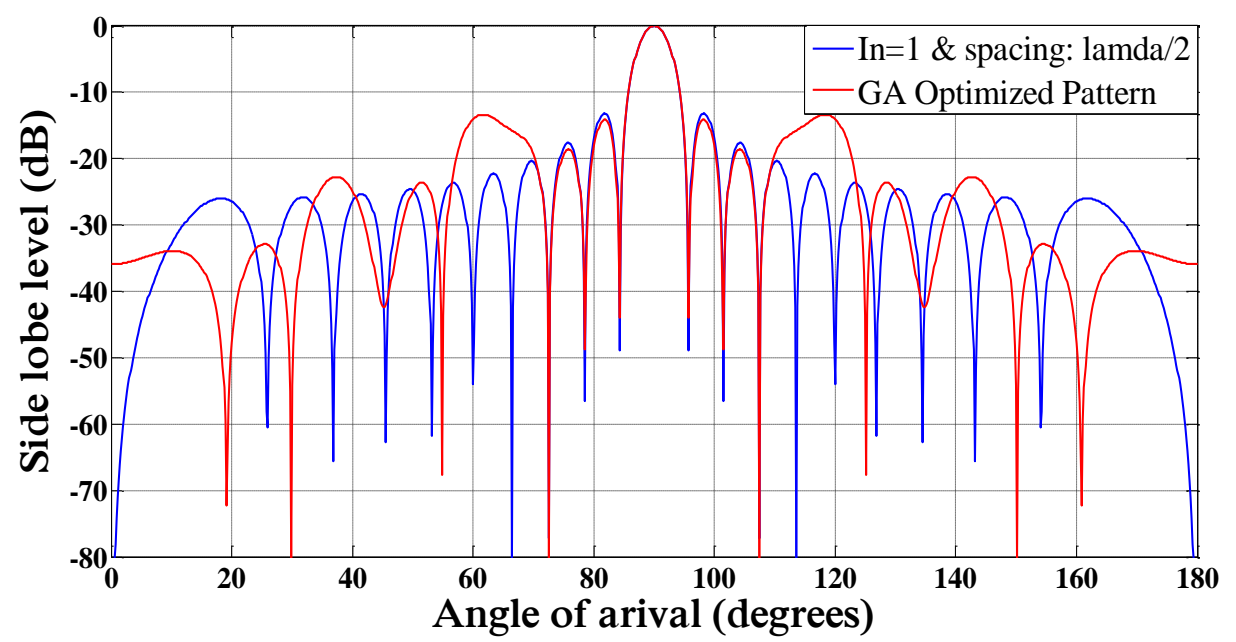

Fig. 6. Best array pattern found by GA for the 20-elements array case with an improved null at 3rd null i.e. $\theta=72.50^{\circ}$ and

$$
\theta=107.50^{\circ} .
$$

table II. Current Excitation Weights, Initial and Final Null Depth for Non-Uniformly Excited Time Modulated Linear Array With Optimal Position of Elements $\left(X_{n}\right)$ From Origin For One Null Imposed In The $3^{\text {RD }}$ Null Position

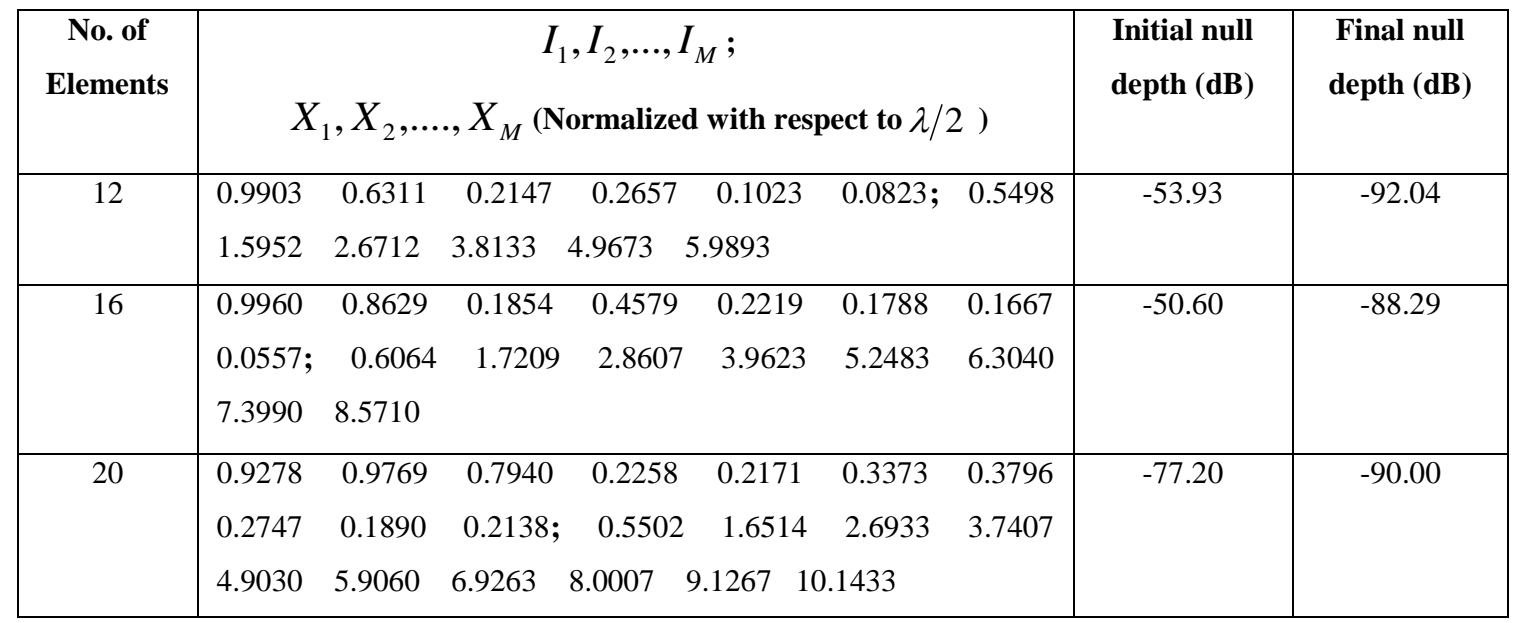

TABLE IIA. SLL and BWFN for Non-Uniformly Excited Time Modulated Linear ArRay With Optimal Spacing $\left(X_{n}\right.$ ) From Array Centre For One Null Imposed at $3^{\text {RD }}$ Null Position.

\begin{tabular}{|c|c|c|}
\hline No. of Elements & SLL Final (dB) & BWFN Final (degree) \\
\hline 12 & -14.27 & 21.02 \\
\hline 16 & -14.51 & 14.40 \\
\hline 20 & -13.36 & 11.52 \\
\hline
\end{tabular}

Figures 7-9 show the imposing of nulls at the $3^{\text {rd }}$ peak for 12, 16 and 20 element structures respectively. For $12,16,20$-element, the nulls have improved up to $-79.60 \mathrm{~dB},-83.17 \mathrm{~dB},-92.00 \mathrm{~dB}$ 
with initial values of $-19.56 \mathrm{~dB},-20.10 \mathrm{~dB},-20.35 \mathrm{~dB}$ respectively. Figures 7-9 also depict the substantial reductions in maximum peak of the SLL with nonuniform current excitation weights and optimal placement of array elements, as compared to the uniform current excitation weights and uniform inter-elements spacing. For example, in optimized pattern the SLL is reduced by more than 1 $\mathrm{dB}$ from $-13.06 \mathrm{~dB}$ down to $-14.57 \mathrm{~dB}$ for 12 elements array. For 16 elements array in optimized pattern SLL is reduced by more than $4 \mathrm{~dB}$ from $-13.14 \mathrm{~dB}$ down to $-17.45 \mathrm{~dB}$. For 20 elements array in optimized pattern SLL is reduced by more than $1 \mathrm{~dB}$ from $-13.19 \mathrm{~dB}$ down to $-14.22 \mathrm{~dB}$. The improved values are shown in TABLE III and TABLE IIIA.

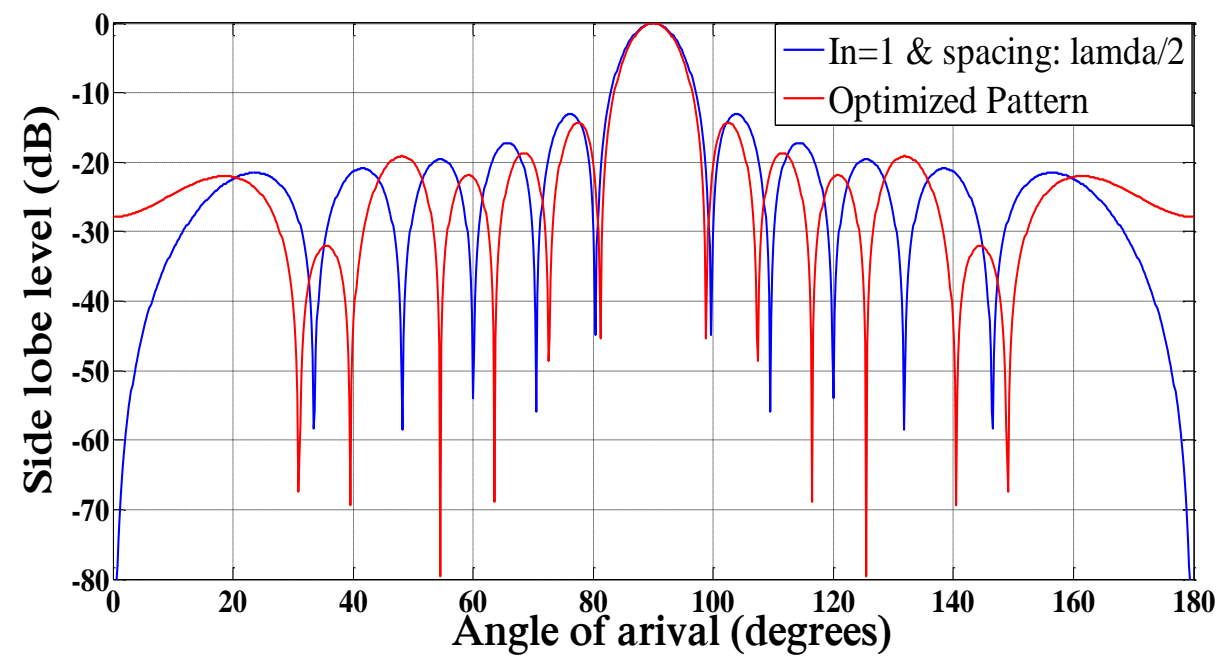

Fig. 7. Best array pattern found by GA for the 12 -elements array case with a null introduced at 3 rd peak i.e. $\theta=54.50^{\circ}$ and $125.50^{\circ}$.

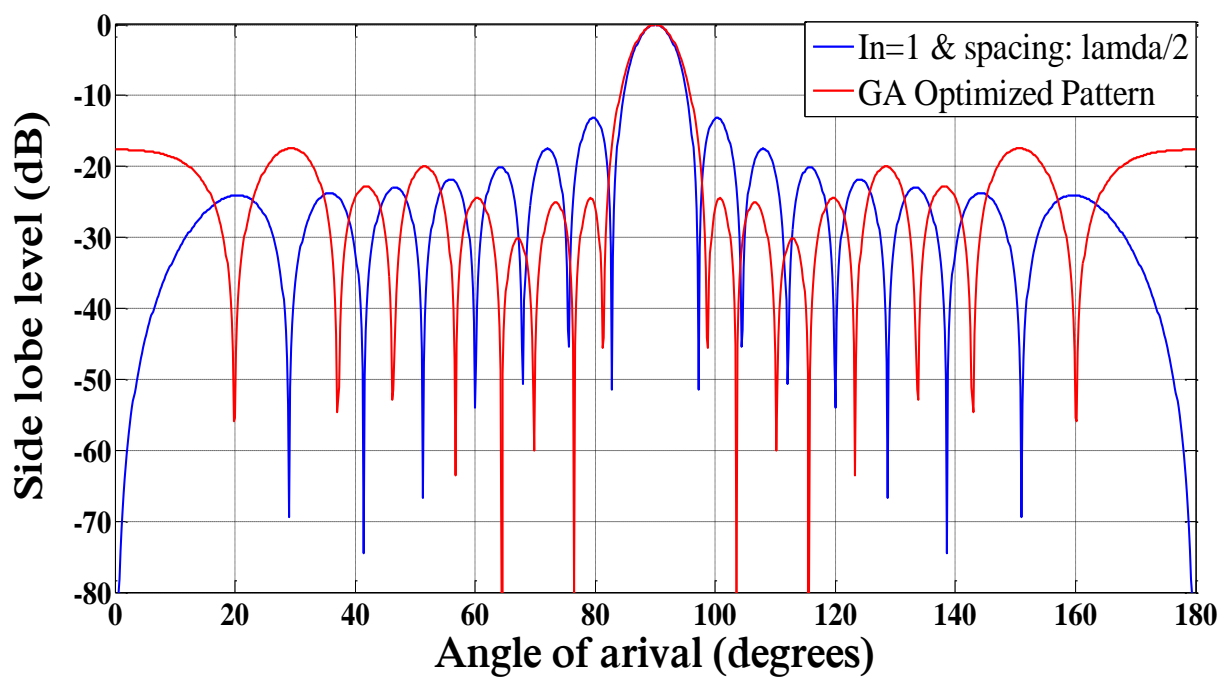

Fig. 8. Best array pattern found by GA for the 16-elements array case with a null introduced at 3rd peak i.e. $\theta=64 \cdot 40^{\circ}$ and $\theta=115.60^{0}$ 


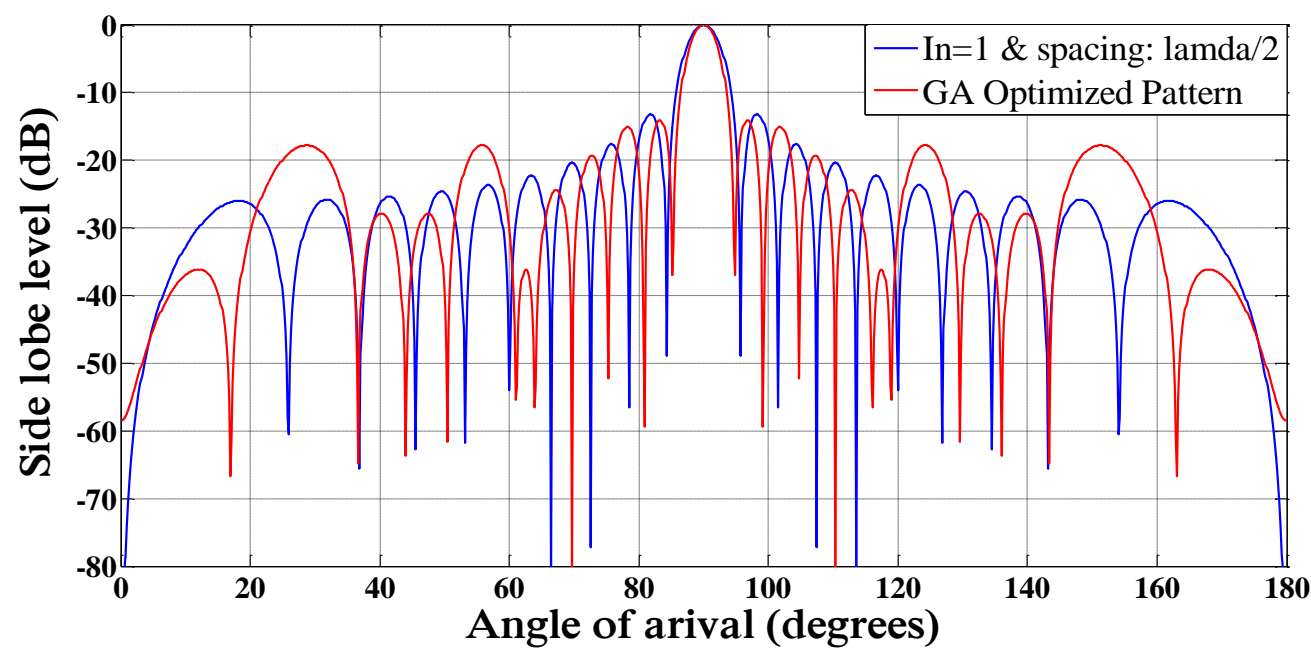

Fig. 9. Best array pattern found by GA for the 20 -elements array case with a null introduced at 3 rd peak i.e. $\theta=69.70^{\circ}$ and

$$
\theta=110.30^{\circ} \text {. }
$$

table III. Current Excitation Weights, Initial Peak and Final Null Depth for Non-Uniformly Excited Time Modulated Linear Array With Optimal Position Of Elements $\left(X_{n}\right)$ From Origin For One Null Imposed In The $3^{\mathrm{RD}}$ PEAK POSITION.

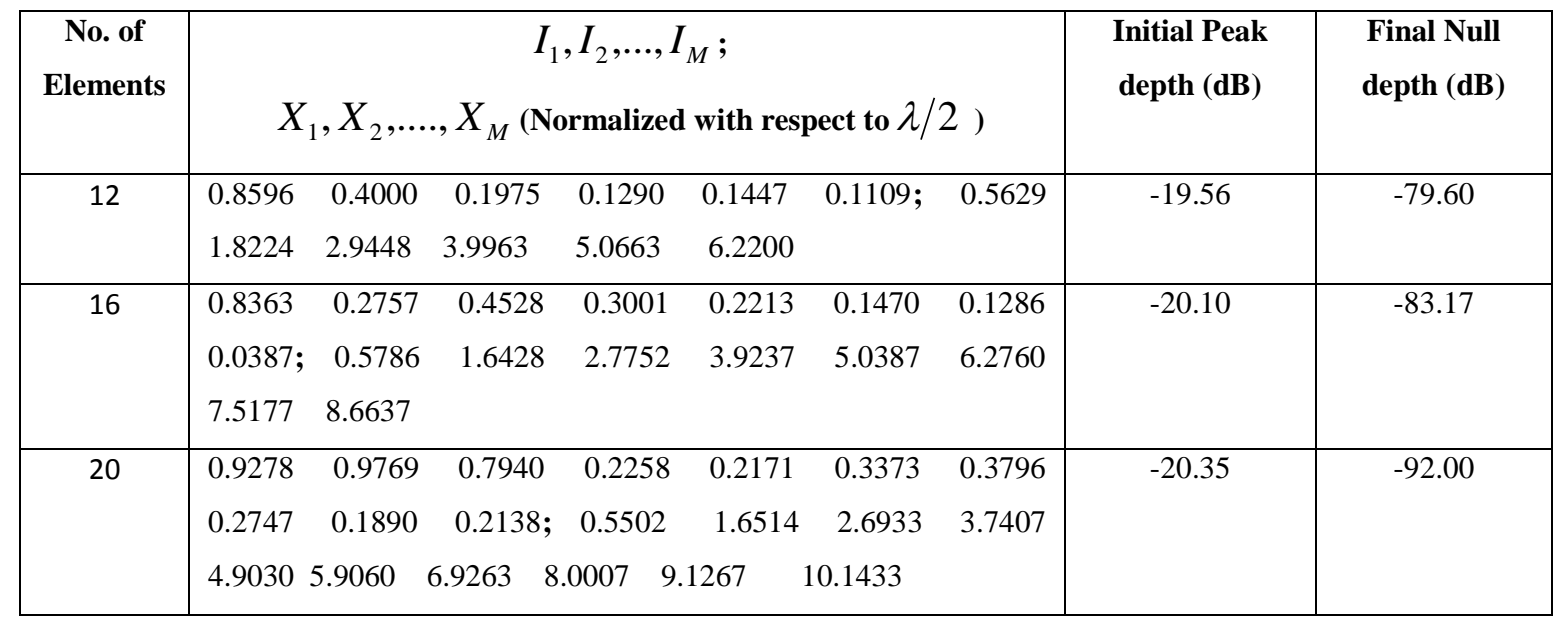

TABLE IIIA. SLL AND BWFN ObTAined For Non-Uniformly ExCited Time Modulated LinEar ARRAy With Optimal Spacing $\left(X_{n}\right)$ From Array Centre For One Null Imposed at $3^{\text {RD }}$ Peak Position.

\begin{tabular}{|c|c|c|}
\hline No. of Elements & SLL Final (dB) & BWFN Final (degree) \\
\hline 12 & -14.57 & 18.00 \\
\hline 16 & -17.45 & 17.10 \\
\hline 20 & -14.22 & 10.00 \\
\hline
\end{tabular}

Figures 10-12 show the imposing of nulls at the $2^{\text {nd }}$ and $3^{\text {rd }}$ peaks for 12,16 and 20 element structures respectively. For 12, 16, 20-element, the pair of nulls has improved up to (-93.32 dB, -102.9 dB), (- 
$91.75 \mathrm{~dB},-72 \mathrm{~dB}),(-71 \mathrm{~dB},-78.92 \mathrm{~dB})$ with initial values of $(-17.22 \mathrm{~dB},-19.56 \mathrm{~dB}),(-17.49 \mathrm{~dB},-20.1$ $\mathrm{dB}),(-17.61 \mathrm{~dB},-20.40 \mathrm{~dB})$ respectively. Figures 10-12 also depict the substantial reductions in maximum peak of the SLL with non-uniform current excitation weights and optimal placement of array elements, as compared to the uniform current excitation weights and uniform inter-elements spacing. For example, in optimized pattern the SLL is reduced by more than $1 \mathrm{~dB}$ from $-13.06 \mathrm{~dB}$ down to $-14.52 \mathrm{~dB}$ for 12 element array. For 16 element array SLL is reduced by more than $1 \mathrm{~dB}$ from $-13.14 \mathrm{~dB}$ down to $-15.05 \mathrm{~dB}$. For 20 element array in optimized pattern SLL is reduced by more than $2 \mathrm{~dB}$ from $-13.19 \mathrm{~dB}$ down to $-15.85 \mathrm{~dB}$. The improved values are shown in TABLE IV and TABLE IVA.

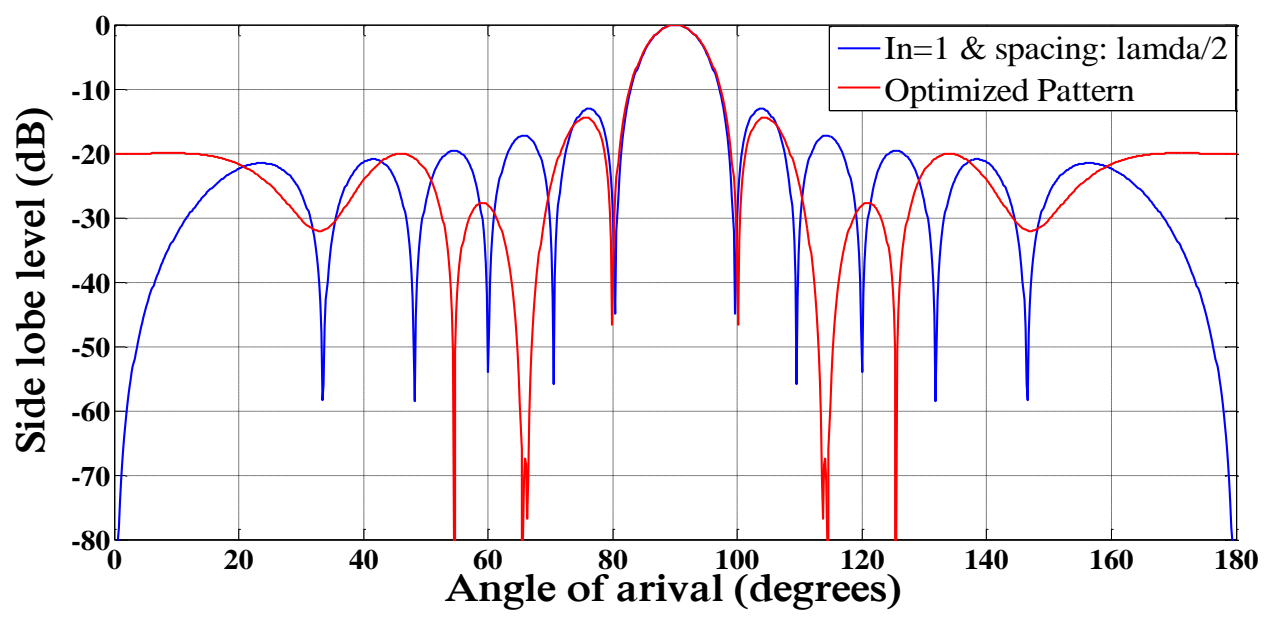

Fig. 10. Best array pattern found by GA for the 12 -elements array case with nulls introduced at $2^{\text {nd }}\left(\theta=65.70^{0}, 114.30^{\circ}\right)$ and $3^{\text {rd }}\left(\theta=54.50^{\circ}, 125.50^{\circ}\right)$ peaks.

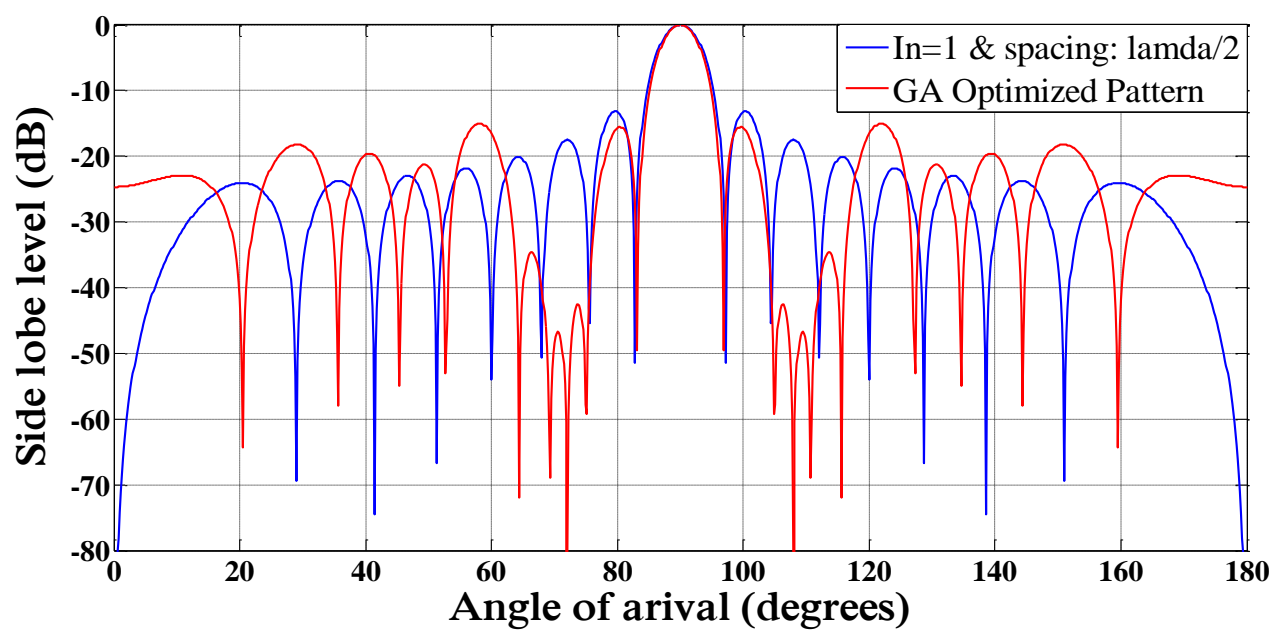

Fig. 11. Best array pattern found by GA for the 16-elements array case with nulls introduced at $2^{\text {nd }}\left(\theta=72.00^{0}, 108^{\circ}\right)$ and $3^{\text {rd }}$ $\left(\theta=64.40^{0}, 115.60^{0}\right)$ peaks. 


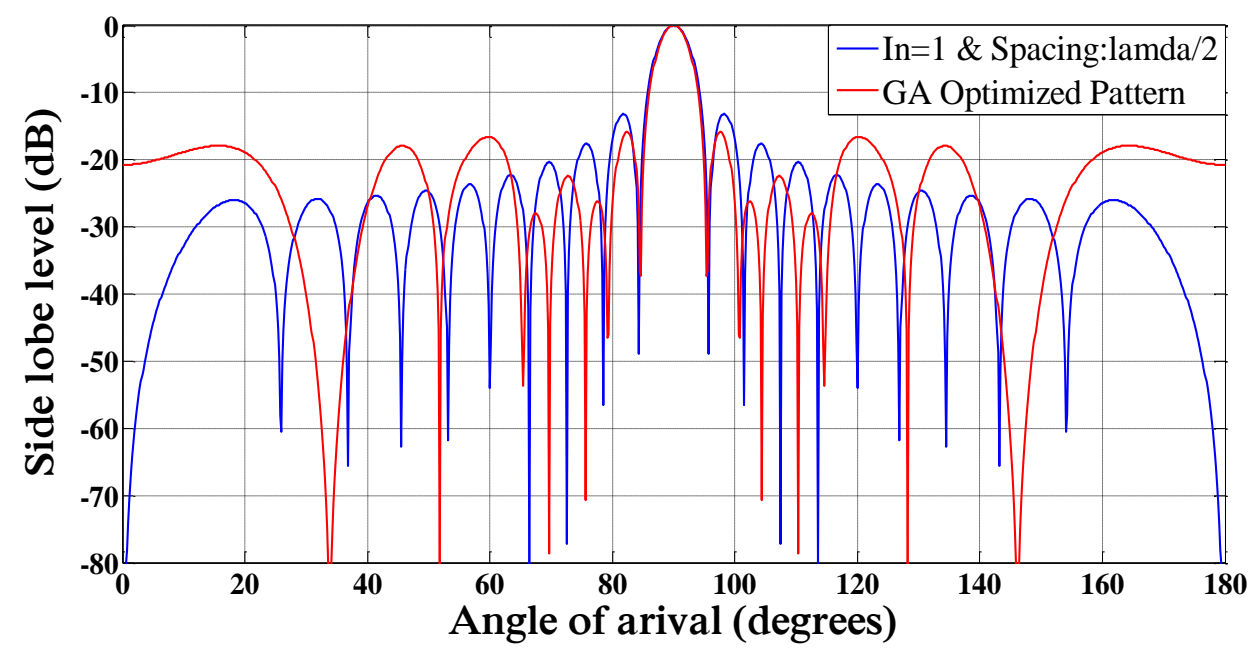

Fig. 12. Best array pattern found by GA for the 20-elements array case with nulls introduced at $2^{\text {nd }}\left(\theta=75.60^{0}, 104.40^{\circ}\right)$ and $3^{\text {rd }}\left(\theta=69.70^{\circ}, 110.30^{\circ}\right)$ peaks.

TABle iV. Current Excitation Weights, Initial Peak and Final Null Depth for Non-Uniformly Excited Time Modulated Linear Array With Optimal Position Of Elements $\left(X_{n}\right)$ From Origin For Nulls Imposed In The $2^{\text {Nd }}$ AND $3^{\text {RD }}$ PEAKS.

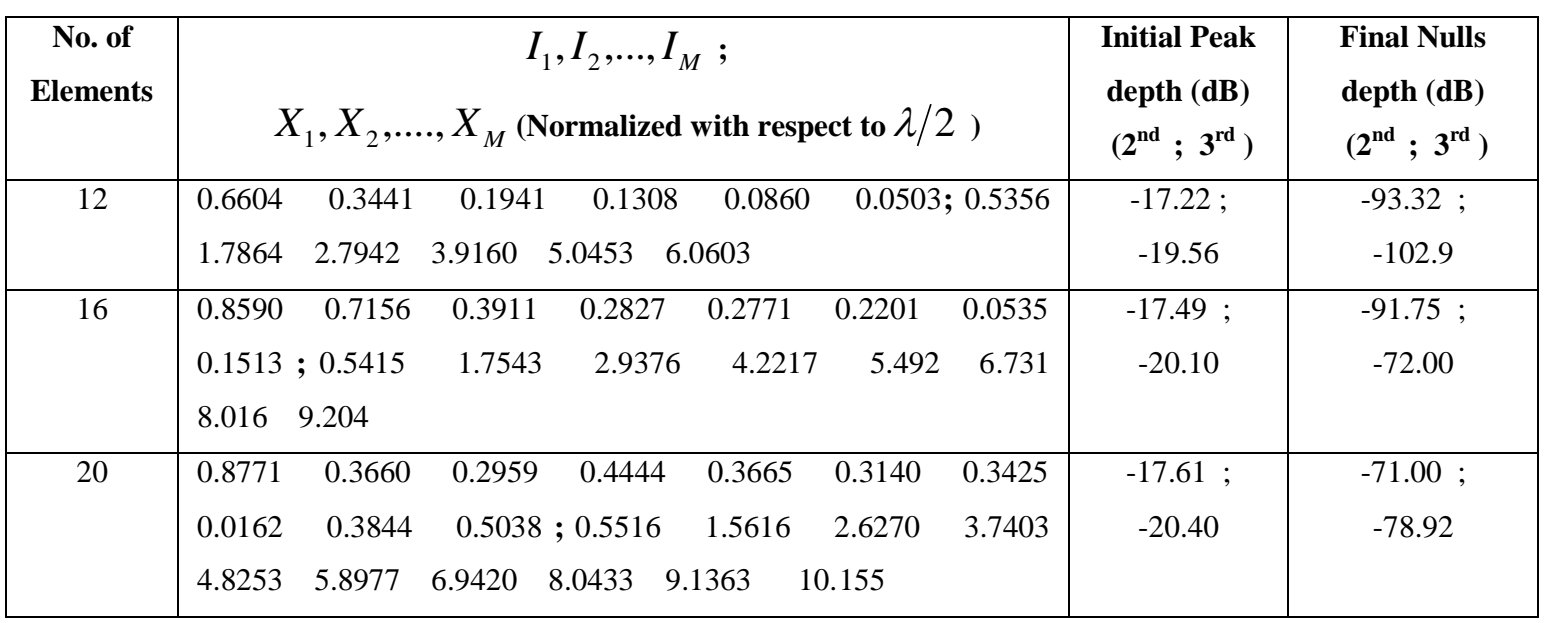

TABLE IVA. SLL AND BWFN fOR NON-UNiformly ExCited Time Modulated LinEar ARRay With Optimal SpaCing $\left(X_{n}\right.$ ) From Array Centre For Nulls Imposed at $2^{\text {ND }}$ And $3^{\text {RD }}$ Peak Positions.

\begin{tabular}{|c|c|c|}
\hline No. of Elements & SLL Final (dB) & BWFN Final(deg) \\
\hline 12 & -14.52 & 20 \\
\hline 16 & -15.05 & 12.20 \\
\hline 20 & -15.85 & 11.52 \\
\hline
\end{tabular}

Figures 13-15 show the imposing of nulls at the $2^{\text {nd }}$ and $3^{\text {rd }}$ nulls for 12,16 and 20 element structures respectively. For 12, 16, 20-element, the pair of nulls has improved up to $(-74.89 \mathrm{~dB},-64 \mathrm{~dB}),(-$ $56.00 \mathrm{~dB},-86.07 \mathrm{~dB}),(-68.60 \mathrm{~dB},-86.36 \mathrm{~dB})$ with initial values of $(-55.83 \mathrm{~dB},-53.93 \mathrm{~dB}),(-45.35 \mathrm{~dB},-$ $50.60 \mathrm{~dB}),(-56.62 \mathrm{~dB},-77.2 \mathrm{~dB})$ respectively. Figures 13-15 also depict the substantial reductions in 
maximum peak of the SLL with non-uniform current excitation weights and optimal placement of array elements, as compared to the uniform current excitation weights and uniform inter-elements spacing ( $\lambda / 2$ spacing between adjacent elements). The improved values are shown in TABLE $V$ and TABLE VA.

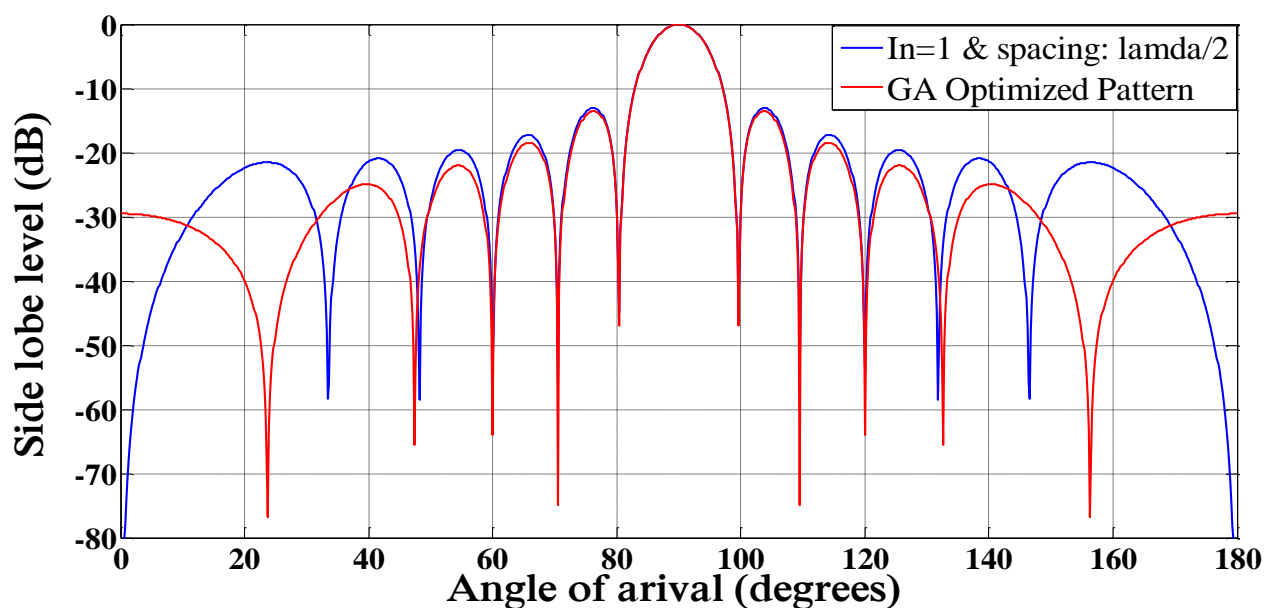

Fig. 13. Best array pattern found by GA for the 12-elements array case with improved nulls at $2^{\text {nd }}\left(\theta=70.60^{0}, 109.40^{\circ}\right)$ and $3^{\text {rd }}\left(\theta=60^{\circ}, 120^{\circ}\right)$ nulls.

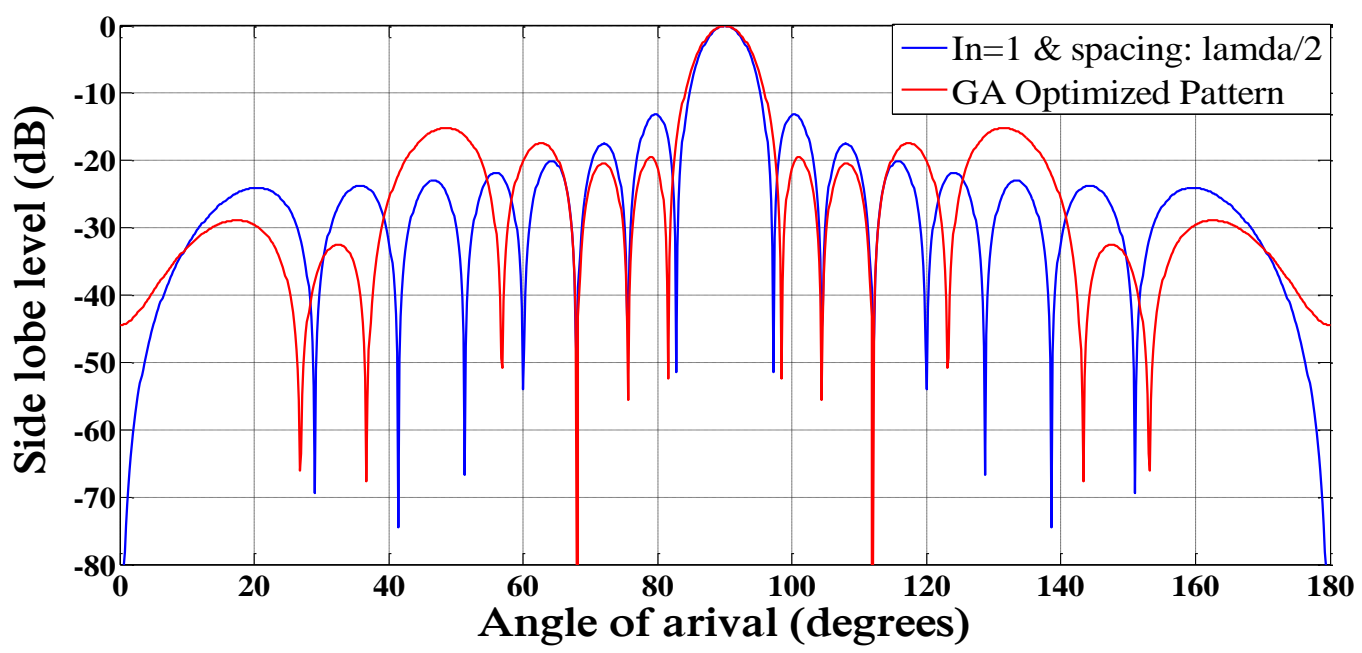

Fig. 14. Best array pattern found by GA for the 16-elements array case with improved nulls at $2^{\text {nd }}\left(\theta=75.60^{\circ}, 104.40^{\circ}\right)$ and $3^{\text {rd }}\left(\theta=68^{0}, 112^{0}\right)$ nulls. 


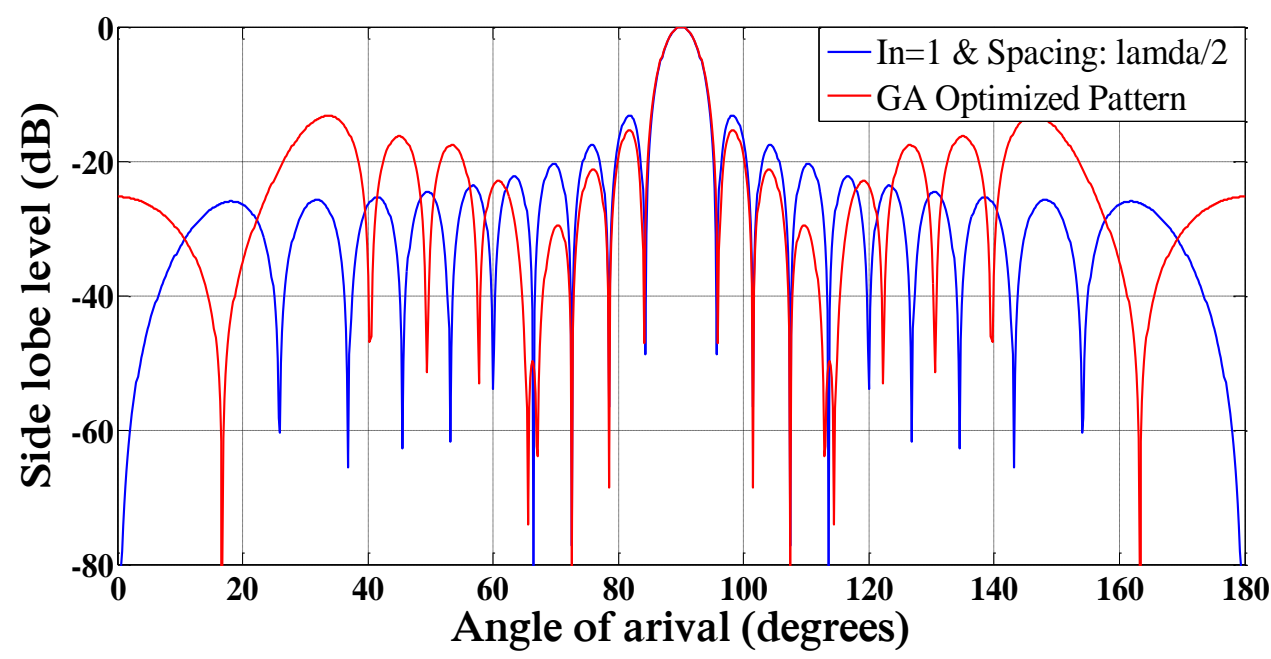

Fig. 15. Best array pattern found by GA for the 20-elements array case with improved nulls at $2^{\text {nd }}\left(\theta=78.50^{\circ}, 101.50^{\circ}\right)$ and $3^{\text {rd }}\left(\theta=72.50^{0}, 107.50^{\circ}\right)$ nulls.

TABle V. Current Excitation Weights, Initial and Final Null Depth for Non-Uniformly Excited Time Modulated Linear Array With Optimal Position Of Elements $\left(X_{n}\right)$ From Origin For Nulls Imposed In The $2^{\text {ND }}$

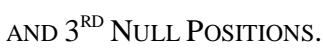

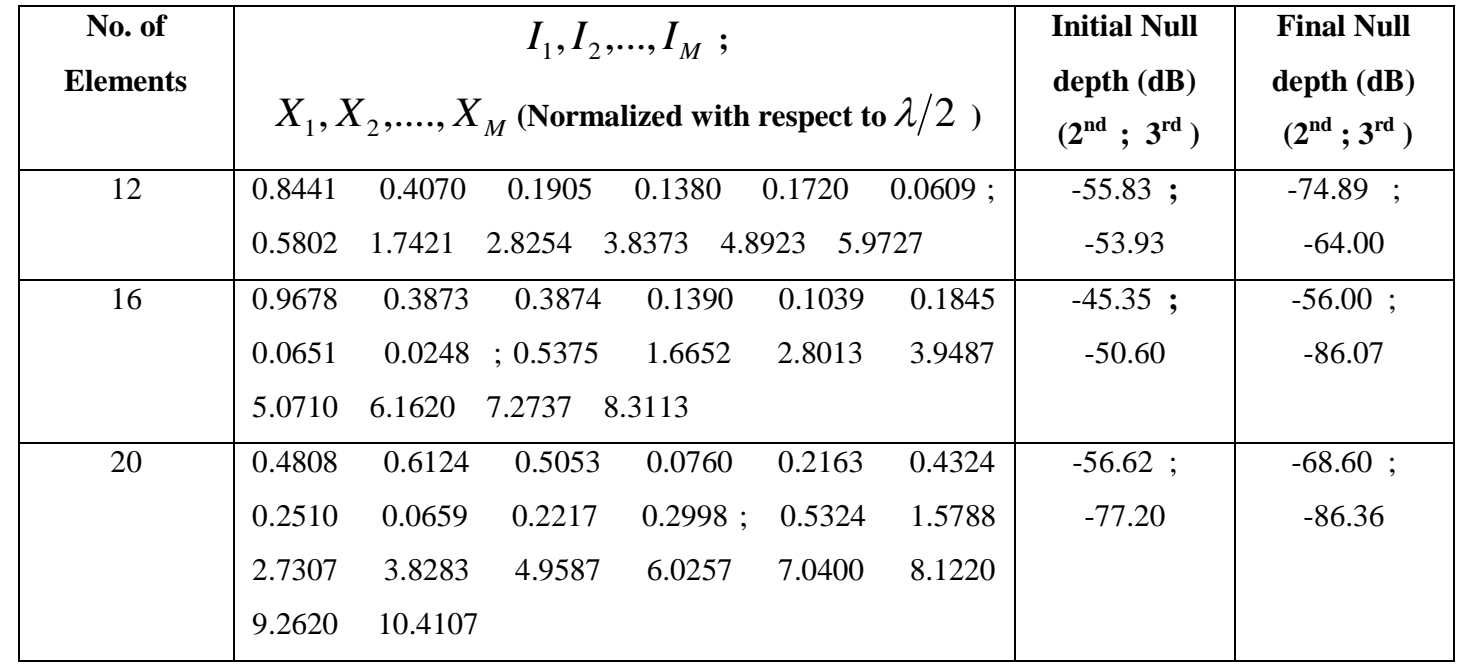

TABLE VA. SLL AND BWFN for Non-Uniformly Excited Time Modulated Linear ARray With Optimal Spacing $\left(X_{n}\right.$ ) From Array Centre For Nulls Imposed at $2^{\text {ND }}$ AND $3^{\text {RD }}$ Null Positions.

\begin{tabular}{|c|c|c|}
\hline No. of Elements & SLL Final (dB) & BWFN Final (deg) \\
\hline 12 & -13.56 & 19.10 \\
\hline 16 & -15.18 & 16.90 \\
\hline 20 & -13.27 & 11.62 \\
\hline
\end{tabular}




\section{CONCLUSIONS}

In this paper, the design of a non-uniformly excited symmetric time modulated linear antenna array with optimized non-uniform spacing between the elements has been described using the optimization technique of Real-coded Genetic Algorithm. Simulated results reveal that optimizing the excitation amplitude of the elements with optimal spacing of the array antenna elements can impose deeper nulls at a desired direction as well as can reduce SLL for a given number of the array elements with respect to corresponding uniformly excited linear array with uniform inter-element spacing of $\lambda / 2$. In almost all the cases nulls depth are improve by more than $-80 \mathrm{~dB}$. On the other hand maximum SLL also reduced in all cases. The BWFN of the initial and final radiation pattern remains approximately same. It is worth noting that, although the algorithm used here is implemented to constrained synthesis for a linear array with isotropic elements, one can see from the proposed technique it is not limited to this case. It can easily be implemented to non-isotropic elements antenna arrays with different geometries for the design of various array patterns.

\section{REFERENCES}

[1] C. A. Ballanis, "Antenna theory analysis and design," 2nd edition, John Willey and Son's Inc., New York, 1997.

[2] Elliott, R. S., “Antenna Theory and Design”, Revised edition, John Wiley, New Jersey, 2003.

[3] R. L. Haupt, and D. H. Werner, Genetic Algorithms in Electromagnetics, IEEE Press Wiley-Interscience, 2007.

[4] R. L. Haupt, "An introduction to gentic algorthim for electromagnetic”, IEEE Anten. Propag. Mag 37(2) pp7-15 April 1995.

[5] J. C. Bregains, J. Fondevila, G. Franceschetti, and F. Ares, "Signal radiation and power losses of time-modulated arrays," IEEE Transaction on antennas and Propagation, vol. 56,no.6,pp. 1799-1804, Jun. 2008.

[6] S. Yang, Y. B. Gan, and A. Qing, "Sideband suppression in time-modulated linear arrays by the differential evolution algorithm,” IEEE Antennas Wireless Propag. Lett., vol. 1, pp. 173-175, 2002.

[7] S. Yang, Y. B. Gan, and P. K. Tan, "A new technique for power-pattern synthesis in time-modulated linear arrays," IEEE Antennas Wireless Propag.Lett., vol. 2, pp. 285-287, 2003.

[8] G. R. Hardel, N. T. Yallaparagada, D. Mandal, and A. K. Bhattacharjee, "Introducing Deeper Nulls for Time Modulated Linear Symmetric Antenna Array Using Real Coded Genetic Algorithm,” IEEE Symposium on Computers \& Informatics (ISCI 2011), pp. 249-254, Kuala Lumpur, Malaysia, 20 - 22 March 2011.

[9] Gang Li, Shiwen Yang, and Zaiping Nie, "Direction of Arrival Estimation in Time Modulated Linear Arrays With Unidirectional Phase Center Motion,” IEEE Transactions on Antennas and Propagation, Vol.58, No.4, pp.1105-1111, April 2004.

[10] M. A. Mangoud and H. M. Elragal "Antenna array pattern synthesis and wide null control using enhanced particle swarm Optimization”, Progress In Electromagnetics Research B, Vol. 17, 1-14, 2009.

[11] Kumar et al., "Ultra-low sidelobes from time-modulated arrays," IEEE Transactions on Antennas and Propagation, vol. AP-11, pp. 633-639, November 1963.

[12] Gopi Ram, D. Mandal, R. Kar, S. P. Ghoshal, "Minimization of Side Lobe of Optimized Uniformly Spaced and nonuniform Exited Time Modulated Linear Antenna Arrays Using Genetic Algorithm", SEMCCO 2012, LNCS Volume 7677, pp 451-458, Bhubaneswar, Odisha, Dec. 20-21, 2012.

[13] G. Ram, D. Mandal, S. P. Ghoshal, R. Kar, "Improved Particle Swarm Optimization Based Side Lobe Reduction in Time Modulated Linear Antenna Arrays", IEEE Indian Antenna Week 2013, Aurangabad, India, 3rd -7th June 2013. 
[14] Goudos, S.K.; Moysiadou, V.; Samaras, T.; Siakavara, K.; Sahalos, J.N.; “Application of a Comprehensive Learning Particle Swarm Optimizer to Unequally Spaced Linear Array Synthesis With Sidelobe Level Suppression and Null Control” Antennas and Wireless Propagation Letters, IEEE, Volume: 9, 125-129, 2010.

[15] D. Mandal, S. P. Ghoshal, and A. K. Bhattacharjee, "Linear Antenna Array Synthesis Using Improved Particle Swarm Optimization,” Second IEEE Int. Conf. on Emerging Applications of Information Technology (EAIT 2011), Kolkata, India, Feb. 19-20, 2011.

[16] D. Mandal, S. P. Ghoshal, and A. K. Bhattacharjee, "Wide Null Control of Symmetric Linear Antenna Array Using Novel Particle Swarm Optimization," Int. Journal of RF and Microwave Computer-Aided Engineering, vol. 21, Issue. 4, pp. 376-382, Apr. 2011.

[17] S. Das, S. Bhattacharjee, A. K. Bhattacharjee, and D. Mandal, "Sidelobe Level and Null Control of Asymmetric Linear Antenna Array using Genetic Algorithm” International Journal Artificial Intelligence \& Computational Research, vol. 2, no. 1, pp. 7-12, 2010.

[18] A. Recioui, A. Azrar, H. Bentarzi, M. Dehmas \& M. Chalal, "Synthesis of Linear Arrays with Sidelobe Level Reduction Constraint using Genetic Algorithms,” International Journal of Microwave and Optical Technology, vol. 3, no. 5, pp.524-530, November 2008.

[19] D. Mandal, S. K. Ghoshal, S. P. Ghoshal, and A. K. Bhattacharjee, "Radiation Pattern Synthesis for Linear Antenna Arrays Using Craziness Based Particle Swarm Optimization" International Journal Artificial Intelligence \& Computational Research, vol. 2, no. 1, pp. 13-18, 2010.

[20] D. Mandal, S. P. Ghoshal, and A. K. Bhattacharjee, "Optimized Radii and Excitations with Concentric Circular Antenna Array for Maximum Sidelobe Level Reduction Using Wavelet Mutation Based Particle Swarm Optimization Techniques,” Telecommunications System, DOI 10.1007/s11235-011-9482-8, 2011.

[21] J.H. Holland, “Adaptation in Natural and Artificial Systems”, Univ. Michigan Press, Ann Arbor, 1975.

[22] Z. Michalewicz, “Genetic Algorithms, Data Structures and Evolution Programs," Springer-Verlag, Berlin, 1999.

[23] Rahmat-Samii, Y. and E. Michielssen (Eds.), Electromagnetic Optimization by Genetic Algorithms, Wiley, New York, 1999. 